\title{
Inteligencia artificial y documentación
}

\author{
Vicente Pablo Guerrero Bote \\ Facultad de Biblioteconomía y Documentación de la U. de Extremadura \\ C/ José María Alcaraz y Alenda, 1 (pasaje) 06011 Badajoz \\ E-mail: vicente@ alcazaba.unex.es \\ Cristina López-Pujalte \\ Facultad de Biblioteconomía y Documentación de la U. de Extremadura \\ C/ José María Alcaraz y Alenda, 1 (pasaje) 06011 Badajoz \\ E-mail: clopez@alcazaba.unex.es
}

Trabajo recibido

el 18 de abril de 2001

*

Trabajo aceptado el 23 de mayo de 2001

\section{RESUMEN}

En la actualidad se están aplicando técnicas de inteligencia artificial a distintos campos de la ciencia para superar los límites de los métodos clásicos; el caso de la documentación no es una excepción. Se presenta un estudio exhaustivo de las distintas aplicaciones que se han dado a las técnicas de IA en este campo. La mayor parte de estas aplicaciones vienen esencialmente de las redes neuronales artificiales, de los algoritmos genéticos (ambos principalmente utilizados en recuperación de información) y de los sistemas expertos (que se aplican más en el ámbito profesional).

Palabras clave: Inteligencia Artificial, Documentación, Recuperación de Información, Redes Neuronales Artificiales, Sistemas Expertos, Algoritmos Genéticos.

\section{ARTIFICIAL INTELLIGENCE AND DOCUMENTATION \\ Vicente P. GuerRero-BOTE Y CRISTINA LóPEZ-PujALTE}

\section{SUMMARY}

Artificial intelligence (AI) techniques are today being applied to distinct fields of science in order to surmount the limitations of classical methods; documentation is no exception. An exhaustive study is presented of the different applications that have been given to AI techniques in this field. For most part these applications are derived essentially from artificial neuronal networks, from genetic algorithms (both used mainly in information retrieval) and from expert systems (applied mainly in professional environments).

Key words: Artificial Intelligence, Information Science, Document Retrieval, Artificial Neural Networks, Expert Systems, Genetic Algorithms. 


\section{INTRODUCCIÓN}

$\mathbf{L}$

a tecnología informática o computacional con algoritmos secuenciales ha superado enormemente a los humanos en algunas tareas, como las operaciones matemáticas. En documentación tal tecnología es ampliamente utilizada para la gestión, almacenamiento y recuperación documental. Sobre la base del modelo booleano, por ejemplo, se gestionan enormes bases documentales con una gran eficiencia y eficacia. Sin embargo existen otras muchas tareas triviales para los humanos, que resultan especialmente complicadas para los ordenadores, tales como el procesamiento del lenguaje natural, el reconocimiento de formas, el proceso de la visión, etcétera. Con este motivo se han desarrollado las técnicas denominadas de InteligeniaA Artificial (IA enlosucesivo).

Los términos intdigeniay artifial son ampliamente utilizados tanto por separado como de forma conjunta, sin embargo, al menos el primero es extremadamente difícil de definir. Tratando de huir de los aspectos filosóficos que estas palabras conllevan, podemos proponer en principio algunas definiciones, no aceptadas universalmente, pero bastante sencillas de comprender:

La inteligencia artificial estudia cómo lograr que las máquinas realicen tareas que, por el momento, son realizadas mejor por los seres humanos." [Rich y Knight 1994]

...la parte de la Informática que trata de Sistemas computerizados inteligentes, es decir, Sistemas que muestran las caractenísticas que pueden asociarse a la inteligencia en lo que se refiere al comportamiento humano: comprensión del lenguaje, aprendizaje, razonamiento, resolución de problemas, etc." [Amat 1989]

El ofrecimiento por parte de la máquina de un comportamiento parecido al humano que es capaz de acomodarse 0 ajustarse a una disposición o situación real o ficticiay poder escoger de acuerdo a una serie de particularidades para dar una respuesta rápida y los más acertada posible. [Costa 1995]

Todas estas definiciones hacen referencia a la idea de lograr un comportamiento inteligente semejante al humano; sin embargo, dentro de la IA también se estudian aquellos problemas que les resultan difíciles incluso a los humanos.

Las motivaciones que han llevado al estudio de esto son: en primer lugar la resolución de los problemas y en segundo la emulación del comportamiento humano. Esta última es más importante de lo que en un principio puede parecer, ya que permite comprender el razonamiento humano para aplicárselo a las máquinas, hacer a éstas comprensibles para los humanos o incluso verificar teorías psicológicas.

Los problemas a los que se aplica la IA son distintos a los solucionados por la ingeniería de software clásica. Mientras los resueltos por esta última son básicamente sistemáticos y sobre procedimientos, y tienen en los datos, procesos y algoritmos su materia prima principal, aquellos a los que se aplican las técnicas de IA se caracterizan por ser eminentemente heurísticos y declarativos. Las tareas que 
históricamente se han abordado pueden clasificarse en tres tipos: las formales, las de la vida diaria y las de los expertos. Las primeras, principalmente relativas a los juegos y a las matemáticas, son las que ocuparon en primer lugar a los científicos, en su intento de hallar o estudiar esa inteligencia que se suponía necesaria para desempeñar tales tareas. Posteriormente, se comenzó a tener conciencia sobre la dificultad que supone para los ordenadores hacer tareas que habitualmente realizamos los humanos, como las derivadas de la percepción, del proceso del lenguaje natural, de la aplicación del sentido común, etcétera. Y por último se han aplicado con bastante éxito a las tareas que realizan los expertos, a quienes se les suponen ciertos conocimientos, como el diagnóstico médico, el análisis molecular, el análisis financiero, la ingeniería, etcétera.

Como indican Rich y Knight [1994], las técnicas de IA tienen tres características principales que las diferencian de los otros métodos:

* La búsqueela para explorar las distintas posibilidades en aquellos problemas donde los pasos a seguir no estén claramente definidos.

* El empleo de conocimiento que permite explotar la estructura y relaciones del mundo o dominio al que pertenece el problema, y la reducción del número de posibilidades por considerar, tal como hacemos los humanos.

* La abstraciónque proporciona la manera de generalizar para los casos intrínsecamente similares.

\section{H istoria}

Aunque se trate de un campo joven, como nos indican Russell y Norvig [1996], la IA hereda diversas ideas, puntos de vista y técnicas de otras disciplinas. D e hecho, dentro de la tradición filosófica han surgido simultáneamente diversas teorías sobre el razonamiento y el aprendizaje, que reducen la visión de la mente a un sistema físico. Dentro de las matemáticas, teorías formales relacionadas con la lógica, la probabilidad, la toma de decisiones y la computación. La psicología ha investigado la mente humana. La lingüística ofrece teorías sobre la estructura y significado del lenguaje. Por último, la informática, ofrece las herramientas.

Son también Russell y Norvig [1996] quienes, a nuestro parecer muy acertadamente, dividen la historia de la disciplina en una serie de fases que hacen bastante comprensible la evolución de ésta:

Génesis (1943-1956): Se puede decir que esta fase comienza con los primeros trabajos de McCulloch y Pitts [1943], neurofisiólogo y matemático respectivamente, quienes idearon un modelo de red neuronal artificial. Y termina con el taller de Dartmouth, en el verano de 1956, donde se establece el nacimiento oficial de la disciplina. En éste resultó llamativo sobre todo el Teórico Lógico de Newell y Simon, programa de razonamiento capaz de realizar demostraciones de teoremas matemáticos. 
* Entusiasminicial, gandesesperanzas(1952-1969). En esta fase los detractores de la disciplina fueron planteando retos que se fueron superando uno tras otro, de ahí que otros autores la denominen la del "Miramamáahorasinmanos". De esta época son llamativos el Sducionador Geneal deProblema (SGP) de Newell y Simon, que a diferencia del TénicoLógicose diseñó para que imitara protocolos de razonamiento humanos, o los programas para el juego de damas de Samuel [1959, 1967]. También florecieron actividades enfocadas a las redes neuronales, Taylor [1956] inicia el estudio sobre las memorias asociativas, Rosenblatt [1958] introduce el Perceptrón para el reconocimiento de patrones, capaz de generalizar, Widrow y Hoff formulan una variante del Perceptrón, la ADALINE (AD A ptative LINear Elements) [Widrow 1960], que fue la primera red neuronal aplicada a un problema real (filtros adaptativos para evitar ecos en las líneas telefónicas), comercializada durante muchos años, así como su extensión MADALINE [Widrow 1962].

* Una dosis derealidad (1966-1974). Propiciadas por los éxitos obtenidos en la etapa anterior, se hicieron previsiones excesivamente optimistas, como más tarde se demostró. Los escollos que se encontraron fueron principalmente dos, en primer lugar, se pensó que la extensión de las técnicas que demostraron funcionar con ejemplos sencillos, pocos objetos, pocas manipulaciones, etcétera, llegarían a los problemas reales con el aumento de la velocidad de cómputo. Sin embargo, estos métodos resultaron demasiado débiles dada la explosión combinatoria que se recibía de la realidad, y a pesar del enorme incremento habido en la capacidad de cálculo. El segundo obstáculo correspondía a las limitaciones de las estructuras utilizadas, un caso particular fue puesto de manifiesto en el libro "Perceptrons" de Minsky y Papert [1969], que aunque solamente afectaba a este tipo de redes supuso la retirada de fondos para las redes neuronales artificiales.

* Sistemas basados en e conoimiento (1969-1979): Para encontrar la solución los programas de la década anterior utilizaban mecanismos de búsqueda junto con pasos de razonamiento elementales. Una forma de superar la explosión combinatoria es emplear conocimiento sobre el dominio del problema para conseguir pasos de razonamiento más amplios. El DENDRAL [Buchanan $\notin$ al. 1969] constituye uno de los primeros ejemplos de este enfoque, diseñado en Stanford para inferir una estructura molecular a partir de la información proporcionada por un espectrómetro de masas. El siguiente esfuerzo de consideración se dio en el área del diagnóstico médico con el programa MYCIN, para el diagnóstico de las infecciones sanguíneas, que con un respaldo de 450 reglas era capaz de hacer diagnósticos tan buenos como los de un experto y, desde luego, mejores que los de un médico recién graduado.

* Seconvieteen una industria (1980-1988): El primer sistema experto comercial, R1, inició sus actividades en Digital Equipment Corporation [McD ermott 
1982]. En 1988, el grupo de inteligencia artificial de la D EC había distribuido ya 40 sistemas expertos, y esperaba tener más. Du Pont utilizaba ya 100 de éstos y estaban en etapa de diseño 500 más, lo que le generaba ahorros de diez millones de dólares anuales. Casi todas las compañías importantes de USA contaban con su propio grupo de desarrollo que utilizaba o investigaba la tecnología de los sistemas expertos. En 1981 los japoneses anunciaron el proyecto de la "quinta generación", un plan de 10 años para construir computadoras inteligentes que ejecutasen Prolog, de manera idéntica a como las computadoras comunes lo hacen con el código de máquina, lo que le devolvió el crédito a la disciplina.

* El despetar delas reedes neuranales (1980 al presente). Tras el libro de Minsky y Papert [1969] se redujo drásticamente la investigación en ese campo, aunque se continuó en otras áreas, como la física. Grossberg [1968] y Amari [1972] introducen independientemente el modelo aditivo de neurona, explorado para el estudio de la memoria a corto plazo, que serviría de base al posterior modelo de Hopfield. También surgieron en esta década de "letargo" los primeros estudios sobre mapas autoorganizativos que utilizaban un aprendizaje competitivo [Malsburg 1973, G rossberg 1972] y dieron pie a Kohonen [1982] para iniciar sus estudios. Anderson presenta el Asociador Lineal y una potente extensión llamada Brain-State-in-a-Box (BSB) [Anderson \& al. 1977], al mismo tiempo que Fukushima desarrolla el Cognitrón [1975] e inicia su investigación sobre el Neocognitrón [1980]. Con esta base en los años 80 resurge el interés por las redes neuronales y se presentan nuevas aportaciones a la teoría y diseño de éstas desde diferentes frentes. En 1982 se celebró la U.S.-Japan Joint Conference on Cooperative/ Competitive Neural Networks y Fujitsu comenzó el desarrollo de computadores pensantes para hacer aplicaciones en robótica.

\section{Estado de la cuestión}

Como hemos podido ver en la sección anterior dedicada a la historia de la disciplina, se trató en primer lugar de resolver una serie de problemas formales para los que se suponía necesaria la inteligencia, y surgieron unas técnicas que los reducían a búsquedas en espacios de estados. D e este modo, los programas tenían que ir navegando por dichos espacios hasta encontrar la solución correcta.

Uno de los métodos de búsqueda más potentes en la IA (y que están adquiriendo más notoriedad en la actualidad) son los Algritmos Genéticos[Holland, 1992; G oldberg, 1989; Michalewicz, 1995]. Los Algoritmos Genéticos (en lo sucesivo AA.G G. en plural y ag en singular) una técnica de IA que utiliza procedimientos adaptativos, están destinados a resolver problemas de búsqueda que satisfagan ciertos requisitos y estén inspirados en los mecanismos de la evolución biológica, además de basarse en el principio de selección natural y de los códigos genéticos de las especies. 
Los AA.G G. tienen como entrada una población de individuos llamadoscromosomas que son generados aleatoriamente y representan las posibles soluciones al problema, o bien, si contamos con algún conocimiento sobre la distribución del óptimo podemos usar esa información para crear parte del conjunto inicial de soluciones potenciales [Michalewicz, 1995; Yang y Korfhage, 1994].

Estos individuos van cambiando (evolucionando) en sucesivas iteraciones llamadas generaiones mediante procesos de seleción, cureymtación. Estas iteraciones terminan cuando el sistema deja de mejorar o cuando se alcanza un número máximo prefijado de generaciones. La salida del AG será el mejor individuo de la población final o una combinación de los mejores cromosomas de ella.

El funcionamiento básico de los AA.GG. es el siguiente: tratar de mantener una población de individuos, cada uno de ellos caracterizado por un código genético que lo caracteriza unívocamente, y simular una evolución de dicha población a lo largo del tiempo, basada en la aparición de nuevos individuos como resultado de cruces, mutaciones y reproducciones de los individuos antiguos. Cada uno de los individuos representa una posible solución al problema y la evolución se realiza de manera que los individuos de cada generación tengan una mayor probabilidad de ser mejores soluciones que los individuos de la generación anterior.

Los AA.G G. tienen un gran campo de aplicación en documentación, en concreto en recuperación de información, ya que constituyen una técnica de búsqueda versátil e independiente del dominio que conlleva implícito un cierto paralelismo, muy adecuado para los enormes espacios a que dan lugar las bases documentales. Así, fueron apareciendo poco a poco investigaciones que empleaban AA.GG. en algún aspecto de la recuperación documental. Muchos investigadores en ciencias de la información comenzaron a experimentar con estas técnicas [Belew, 1989; G ordon, 1988a, 1988b; Know, 1989, 1990; Raghavan y Agarwal, 1987]. Sin embargo, se puede decir que la investigación que une computación evolutiva con RI es incipiente y está todavía en desarrollo.

Aunque éstos no son específicamente algoritmos de aprendizaje como ocurre con las redes neuronales, sí se pueden emplear en muchas tareas de aprendizaje dado su carácter adaptativo, donde las posibles soluciones evolucionan hacia mejores soluciones, y se acercan al óptimo. Además, si hacemos una comparación de los AA.G G. con los algoritmos de búsqueda tradicionales se observa que los genéticos tienen las siguientes ventajas [Michalewicz, 1995]:

* Los AA.gG. buscan a partir de una población de puntos, no de un único punto [Goldberg, 1989]. De este modo, evitan quedar atrapados en un óptimo local del espacio de búsqueda cuando pueda existir una solución global mejor en algún otro sitio.

* Los AA.G G . utilizan información de lo bueno que son los individuos mediante el uso directo de la función objetivo, ya que hay que señalar que el AG no es una búsqueda aleatoria de una solución a un problema, sino que esta búsqueda, 
esta evolución, está guiada por el grado de adaptación de los individuos de la población [Holland, 1992].

* Los AA.g G. usan reglas de transición probabilísticas, no reglas determinísticas.

Por otro lado, en esa primera época se supuso que se podrían resolver problemas más cercanos a la realidad a medida que fuera aumentando la potencia de cálculo. Sin embargo a pesar del aumento de ésta, en muchos problemas resultaron métodos excesivamente débiles ante la explosión combinatoria que se recibía de la realidad (que implicaba enormes espacios de estados).

La mera observación de los humanos permitió ver cómo hemos aprendido ciertas reglas que evitan que tengamos que considerar todas las posibilidades, y que por tanto reducen enormemente el espacio de búsqueda. D e ahí algunas definiciones del la IA como por ejemplo:

Estudio de las técnicas de resolución de problemas de complicación exponencial en un tiempo polinomial mediante el uso de conocimiento sobre el campo. [Rich y Knight 1994]

Esto dio lugar a un tipo de sistemas que son los llamados Sistemas Basados en Conoimientoo SistemasdeConoimientos Este calificativo se podría aplicar a cualquier programa que funcione bien, sin embargo, mientras el resto solamente contiene conocimientos relativos a los procedimientos que implementan, éstos, además, operan directamente con conocimientos.

Siguiendo a Gómez đal. [1997], los SS.BB.CC. suelen tener una estructura común cuyos elementos básicos son:

a) La BasedeConoimientos que contiene los conocimientos relativos a la tarea. Se utilizan formalismos de representación para codificar los conocimientos en ella.

b) El Motor deInferenias que es el medio por el cual se controla y aplican los conocimientos. Los mecanismos de inferencia permiten que el sistema razone a partir de los datos, noticias y conocimientos de entrada para producir los resultados de salida.

c) Intefaz deEntrada/ Salida, que permite al usuario proporcionar hechos y datos, y al sistema realizar preguntas y proporcionar consejos y explicaciones. En ocasiones, los usuarios y/ 0, expertos pueden interaccionar desde esta interfaz con la base de conocimientos para introducir algunos nuevos.

Un tipo particular de SS.BB.CC. lo forman los que se ha dado en llamar Sistemas Expatos(SS.EE. a partir de ahora), que son aquellos cuyas prestaciones intentan rivalizar con los expertos humanos. En otros términos, son programas que aplican conocimientos substanciales de áreas específicas de la experiencia humana a la solución de problemas, y que poseen como dato conocimiento humano consistente en hechos y heurísticas sobre una tarea o un dominio [Hayes-Roth, e. al., 1983; 
Hayes-Roth y Wecker 1992]. En general, estos SS.EE. tienen un dominio de uso menos amplio y de menor flexibilidad que los expertos humanos (por esta razón, a veces, se les tacha de poco realistas).

Para ello se necesita almacenar esas reglas o ese conocimiento de forma que se pueda razonar con ellas. La manera tradicional de representarlo y procesarlo es la que marca la lóǵca. Podemos decir que la lógica data de tiempos de Aristóteles. Para poder representar un mundo con un cierto nivel de detalle no basta con la lógica de proposidiones hay que utilizar la de predicados Sin embargo, resulta difícil mediante la lógica tratar relaciones, representar lo expresado mediante el lenguaje natural; incluso es muy costoso pasar de lo general a lo particular. Uno de los modelos que surgieron como respuesta es el de las reeles asociativas, dentro de las cuales podemos encontrar las redes semánticas, especialmente aptas para procesar el lenguaje natural, o las redes bayesianas, para representar las relaciones causales, muy utilizadas en la diagnosis médica. El último de los modelos surgidos es el de los marcosde Minsky, llamados así por traducción de la palabra inglesa frames aunque parece más acorde el término plantilla. De forma similar han surgido los guiones para representar situaciones determinadas.

No obstante, como indican Alexander \&.al. [1986], la creación de estos sistemas depende demasiado de la recolección informal de conocimiento humano y de su representación en esquemas adecuados para el procesamiento simbólico. Esto unido a que a finales de los ochenta se aceptaba que era necesaria una conceptualización sobre el conocimiento del dominio [Scott $\&$ al. 1991] hizo que adquiriera gran notoriedad el concepto de ontología que ya había utilizado Sowa en [1984] (el término ontología viene del campo de la filosofía donde se define como la rama de la filosofía que se ocupa de la naturaleza y organización de la realidad). La definición declarativa más consolidada es la que describe una ontología como una especificación explícita de una conceptualización [Gruber, 93]; es decir, que proporciona una estructura y contenidos de forma explícita y que codifica las reglas implícitas de una parte de la realidad; estas declaraciones explícitas son independientes del fin y del dominio de la aplicación en el que se usarán o reutilizarán sus definiciones. La gran diferencia con el resto de las bases de conocimiento es el propósito de la codificación de su conocimiento: las ontologías son diseñadas con suficiente abstracción y generalidad como para compartir y reutilizar el conocimiento [Guerrero y Lozano 1999]. D e este modo, existen servidores que almacenan ontologías de ciertos campos del conocimiento, así como estándares que les permiten a unos sistemas dialogar con otros intercambiando información.

O tro tipo de sistemas que ofrecen un comportamiento inteligente del que apenas hemos hablado, lo forman las redes neuronales artificiales (en lo sucesivo RR.NN.AA. en plural y RNA en singular). Éstos nacen con la idea de superar, por un lado, los algoritmos secuenciales que tan eficientes se han mostrado en tareas de cálculo pero tan ineficientes en tareas "intdectuales" sencillas para los humanos como 
el reconocimiento de imágenes, el procesamiento del lenguaje, etcétera. Y por otro lado se proponen sobrepasar la arquitectura de cálculo convencional de Von Neuman.

Estos sistemas han sido definidos a lo largo de estos años de la siguiente forma:

- ... un sistema de computación hecho por un gran número de elementos simples, elementos de proceso muy interconectados, los cuales procesan información por medio de su estado dinámico como respuesta a entradas externas [Hecht-Nielsen 1988].

- Las redes neuronales artificiales son redes interconectadas masivamente en paralelo de elementos simples (usualmente adaptativos) y con organización jerárquica, los cuales intentan interaccionar con los objetos del mundo real del mismo modo que lo hace el sistema nervioso biológico [Kohonen 1988].

- Son un modelo matemático compuesto por un gran número de elementos procesales organizados en niveles [Hilera 1995].

- Es una nueva forma de computación, inspirada en los procesos biológicos [Hilera 1995].

Son una forma de acometer algunos problemas (algunos de los cuales inabordables de otro modo). Llevan asociado un entrenamiento, mediante el cual se adaptan al problema, y un gran tiempo de procesamiento. Sin embargo, este procesamiento es masivamente paralelo y puede llevarse a cabo sobre estructuras de computación que vienen a ser la implementación física de dichas redes neuronales. Por todo ello, podemos decir que son tanto una serie de algoritmos o formas de abordar problemas, como unas estructuras de computación.

El cerebro está formado por unas células nerviosas llamadas nuronasque tienen una pequeña capacidad de procesamiento. Estas están fuertemente interconectadas a través de las ramas de salida (axones) que producen sinapsis con las ramas de entrada (dendritas) de otras. En el sistema nervioso humano existen cerca de $10^{11}$ neuronasy di ardende $10^{15}$ interconexiones. Las redes nerronalesartificialesnacen por el interés de emular el funcionamiento del cerebro mediante pequeños elementos de procesamiento fuertemente interconectados entre sí, de ahí el nombre.

De forma más general podemos hablar de modelos conexionistas, los cuales, a diferencia de las redes neuronales, no tratan de emular directamente el funcionamiento del cerebro, aunque, llevan a cabo sus tareas mediante elementos de procesamiento fuertemente interconectados.

Estos modelos se llaman también subsimbólicos [Rich y Knight 1994], porque no manejan símbolos, y superan a los simbólicos en algunas tareas de bajo nivel, como reconocimiento de imágenes, clasificación, etcétera. Como ya hemos dicho implican una fase de entrenamiento durante la cual aprenden de ejemplos, y crean y organizan su propio conocimiento, que más tarde explotan en la fase de producción. La diferencia es que este conocimiento está depositado en los pesos sinápticos de la red, lo que hace difícil su extracción y comprensión para los humanos. 
Como hemos dicho anteriormente, tras la publicación del libro "Peceptrons" de Minsky y Papert [1969], la ciencia de la computación detuvo la investigación en esta área; sin embargo, se continuó trabajando en otros campos como la física. $\mathrm{Al}$ resurgir, la investigación en inteligencia artificial estaba en un momento delicado, se habían dado cuenta que para construir un SE satisfactorio hacía falta algo más que un sistema de razonamiento que estuviera lleno de reglas. Algunos anticiparon la llegada de un "inviemo", acompañado de una severa reducción de fondos. Q uizá este temor, así como los factores históricos que han caracterizado el área de las redes neuronales, dieron como resultado que durante una época redes neuronales e inteligencia artificial tradicional se vieran como rivales entre sí. Incluso en el estudio de Van der Besselaar y Leydesdorff [1997] podemos comprobar cómo sus entornos de citas son diferentes. Sin embargo, no son más que campos que aportan enfoques complementarios y coadyuvantes en la solución de un mismo tipo de problemas.

\section{Utilización en documentación}

Como ya expusimos, dentro de la disciplina objeto del estudio tienen cabida tanto los AA.G G., como lo que se conoce como SS.BB.CC., un tipo particular de los cuales son los SS.EE., y las RR.NN.AA., los que tienen una mayor actualidad. En este apartado veremos una colección de proyectos en los que se han aplicado estas tecnologías a la documentación.

Respecto a los AA.G ., y siguiendo a Cordón đ. al. [1999], existen tres grandes áreas de aplicación de los AA.G G. dentro de la RI:

* Indización de documentos

* Agrupamiento o clustering

* Mejoras en la formulación de consultas.

Las aplicaciones que se engloban dentro del primer grupo, el de indización de documentos, se encuentran dirigidas al aprendizaje u optimización de las descripciones de los documentos existentes en una base documental con el fin de mejorar la recuperación de éstos ante una consulta. O ptimización que algunas veces versa sobre la selección de los términos que describen los documentos, y otras sobre sus pesos asociados. D estacan dentro de este grupo el experimento de G ordon [1988a] y el de Vrajitoru [1998].

Como solución a las diferencias existentes entre consultas de distintos usuarios, Gordon propone que no haya una descripción única para cada documento sino que cada documento tenga asociado un conjunto de descripciones y que éstas evolucionen a lo largo del tiempo. Por lo tanto, la población inicial del AG estará formada por diferentes descripciones de un mismo documento, que competirán entre sí por representar al documento de la mejor forma posible. Investigaciones previas confirmaban la eficacia de tal adaptación [G ordon, 1985]. 
En cuanto al trabajo de Vrajitoru, el enfoque es distinto, se quiere extender el modelo de G ordón para que cubra a toda la colección. En la aplicación de G ordon la población está formada por descripciones del mismo documento, y para obtener las mejores descripciones de todos los documentos que conforman la población es necesario ejecutar el algoritmo tantas veces como documentos contenga ésta. Por el contrario, en este trabajo, para incluir todos los documentos de la colección, cada cromosoma codifica la indización de la colección completa de documentos, concatenando en el mismo vector las descripciones de los distintos documentos.

D entro del segundo grupo, el referido a agrupamiento o clustering, se observan dos tipos de aplicaciones: las aplicaciones que realizan clustering de términos [Robertson y Willet, 1995], y las que realizan clustering documental [G ordon, 1991; Raghavan y Agarwal 1987].

En el trabajo de Robertson y Willet se propone un AG para agrupar los términos de tal modo que la suma de las frecuencias de aparición en la colección de documentos sea aproximadamente igual en cada grupo. Para los autores, esta aplicación tiene varias utilidades, por ejemplo: la generación de cadenas de bits para búsquedas por signatura (text-signature searching), la compresión de textos en lenguaje natural, la indización o la clasificación de diccionarios.

En el trabajo de Gordon [1991], el verdadero propósito del AG era cambiar la descripción de los documentos para mejorar la recuperación, y los experimentos revelaron que se podía generar un agrupamiento o clustering orientado al usuario. Gordon vio que el algoritmo era fácilmente convertible en un algoritmo de agrupamiento basado en densidad [Everitt, 1980]. La fuerza de estos métodos es que producen intuitivamente llamativos "clusters naturales" : una región altamente densa de puntos separada por regiones menos densas de otros clusters. Sin embargo el autor no logró asociar correctamente las preguntas de usuario y los clusters.

Raghavan y sus colaboradores también han estudiado los AA.GG. aplicados al clustering de documentos con varios fines. En uno de sus trabajos lo que perseguían era obtener el clustering óptimo [Raghavan y Birchard, 1979] y, más recientemente [Raghavan y Agarwal, 1987] hicieron un estudio para determinar los puntos fronterizos para el algoritmo de clustering adaptativo de Yu \&. al. [1985], que realiza un clustering orientado al usuario.

Por último, el tercer grupo de aplicaciones de mejoras en la formulación de consultas es el más numeroso. Todos los trabajos incluidos en este grupo tienen en común el empleo de un AG para realizar la técnica de retroalimentación por relevancia y, según lo que abarque el aprendizaje adaptativo del AG , las distintas aplicaciones realizarán un aprendizaje u optimización de términos (Chen [1995] y colaboradores [1998b y 1998c]), de términos y pesos (Robertson y Willet [1996], Yang y Korfhage [1994], Horng y Y eh [2000], Martin-Bautista y colaboradores [1999 y 2000] y López-Pujalte [2000]), o consultarán al completo, es decir, términos, pesos 
y operadores booleanos, aunque hay que decir que en este último caso no se trata de AA.GG . propiamente dichos, sino de otra variante de Algoritmo Evolutivo denominada Programadón Genática (Kraft y otros [1997], Sánchez y otros [1995], y Smith y Smith [1997]).

En cuanto a los SS.BB.CC., es necesario aclarar que, como se trata de la aplicación a un campo profesional, se ha tenido principalmente en cuenta el subconjunto que forman los SS.EE., los cuales, como hemos dicho en apartados precedentes, se caracterizan por poder rivalizar con los expertos humanos y ser, por tanto, los que se pueden aplicar a un campo real.

A pesar de la existencia de mucha literatura sobre este tipo de sistemas en biblioteconomía y documentación (ByD en lo sucesivo), uno puede preguntarse si dicho dominio es apropiado para aplicarle esta tecnología. Para ser adecuado, un dominio debe tener claramente circunscritas las fronteras, y contener un número limitado de entidades y relaciones [Sowa 1984; Welbank 1983]. Con respecto a sólo un subdominio de la ByD, la recuperación de la información, Brooks afirmó:

El mundo de la R. I. inteligente resulta ser muy amplio, incluyendo como debe usuarios y subproblemas, documentos y sus descripciones, y heurísticas de recuperación. En otras palabras el dominio no es reducido, ni discreto, ni homogéneo y contiene una gran cantidad de objetos y relaciones. [Brooks 1987]

Sparck-Jones [1987] manifiesta estar igualmente de acuerdo. Así, no sorprende que ningún SE cubra el rango de conocimientos y experiencia que un profesional de la ByD debería poseer.

Entre los 139 SS.EE. contabilizados por Poulter \& al. [1994] el 80\% de los proyectos estaban destinados a subdominios de la ByD. Éstos se pueden desglosar en un 32\% para trabajos de referencia, un 28\% para recuperación en línea, un $12 \%$ para indización, catalogación o clasificación, 7\% para aplicaciones de gestión de bibliotecas, y el 1\% para resumen. D entro de cada subdominio, los sistemas tienden a restringirse a otros incluso más limitados para evitar problemas asociados con límites mal definidos, o que tienen un gran número de entidades y relaciones.

El sistema PLEX US [Vickery y Brooks 1987] está limitado al tema de jardinería y al grupo de clientes de las bibliotecas públicas, mientras que PO INTER [Smith 1989] está limitado a las publicaciones del gobierno norteamericano.

La modalidad de un sistema (por ejemplo si tutoriza, aconseja, o lleva a cabo una tarea sin ayuda) añade otra categorización. La mayoría de los contabilizados en el mencionado estudio ofrecen la modalidad de "consjo". Por ejemplo Ercegovac [1990] describe el sistema MAPPER, que aconseja a los catalogadores principiantes de mapas. Mientras que estos sistemas son ideales para tratar con materiales que, como los mapas, requieren experiencia en catalogación especializada, no lo son 
para muchos otros materiales. Poulter \&al. encuentran que un 69\% de los sistemas aconsejan solamente, y el 3\% tutorizan. El 9\% puede funcionar en ambos modos, y el 19\% en otros modos. REFSIM puede funcionar en varios modos [Parrott 1989] y ofrecer un modo (Socrático) de consulta, de entrenamiento y de tutoría.

De igual modo, algunas áreas tienen una mayor actividad como son las de referencia y los intermediarios "online”, aunque, cada vez se emplean en más áreas (en gran medida influidas por la potencia creciente de los PC y la sofisticación del software).

En la Universidad de Houston se instaló un sistema experto para referencia [Gunning 1992] que pasó de 229 usos en el primer mes a 1749 al final del tercer mes. Llegó a ser tan popular que los usuarios evitaban utilizar fuentes impresas.

AquaRefes un sistema experto diseñado por la Biblioteca Nacional de Agricultura de EE.UU. para ayudar en un rango limitado de preguntas frecuentes sobre piscicultura [Hanfman 1989]. Se realizaron 600 peticiones del programa en los primeros nueve meses de su distribución.

El Patent Infomation Assistant [Ardis 1990] fue desarrollado conjuntamente por dos programadores y dos bibliotecarios especializados en referencias de patentes. D urante dos años ha sido utilizado 83 horas a la semana; nunca estaba cansado, irritado, ofrece información más adaptada a los usuarios que los expertos humanos, y viene muy bien en ciertas peticiones confidenciales.

El GiftA ssistant determina si la biblioteca debe aceptar donaciones particulares y se asegura de que las donaciones de mayor valor sean procesadas más rápidamente [Debrower y Jones 1991].

ORFEO (OnlimeRferencefor Expetisein Opera) recomienda fuentes para hallar información sobre ópera. Contiene una base de conocimiento desarrollada a partir de una importante bibliografía de 700 registros más otros añadidos por Gerber [1992].

También es cada vez más frecuente, en el caso de la recuperación de información, encontrar al menos un capítulo en los manuales recientes, que trate sobre la aplicación de sistemas inteligentes, como es el caso de los manuales de Ellis [1990, 1996]. En concreto, Ellis le da especial importancia a la aplicación de modelos conexionistas y al procesamiento del lenguaje natural como son los proyectos IOTA [Bruandet 1989; Chiaramella y D efued 1987], SCISO R [Rau 1987; Rau \&. al. 1989; Jacobs y Rau 1988, 1990], RESEARCHER [Lebowitz 1986, 1988]. Se pueden encontrar sucesivas revisiones al respecto en [D oszkocs 1986; Smith 1987; Warner 1987; Smeaton 1992 y Haas 1996].

Como tendencias, Poulter \&. al. [1994] apuntan la inclusión de algunos SS.EE. en sistemas de información más amplios, los "emptrados", los cuales están posibilitando la creación de interfaces en lenguaje natural. También indican que la buena voluntad de los profesionales de la información, su experiencia en el área, y su conocimiento de los SS.EE. están ayudando a resolver los problemas. 
En cuanto a las redes neuronales, éstas han sido utilizadas en más de una ocasión y con más de un fin. Siguiendo a Moya \&. al. [1998a] y a Scholtes [1995], podemos decir que las RR.NN.AA. se han aplicado en la ByD a la gestión bibliotecaria, la clasificación de información, el diseño de interfaz, el filtrado de información, la búsqueda incompleta y el descubrimiento inteligente de información (Datamining).

A veces incluso se ha representado al procesamiento de recuperación documental como una red de tres capas, que tiene como entrada la query(pregunta) y como salida los documentos recuperados, mientras que la capa oculta representa de algún modo a los términos indizadores. D oszkocs \&. al. [1990] opinan que esto es definido implícitamente por algunos autores [Bush 1945; D oyle 1961, Salton 1981, Sparck-Jones 1971, Stiles 1961]. Belew fue uno de los primeros en perfilar una red de este tipo [1989] y Kwok la utilizó con un aprendizaje Hebbiano modificado [1989, 1990].

Wilkinson y Hingston [1991, 1992] han empleado la medida del coseno en un modelo similar para recuperar información. Recientemente, Cortez et. al. [1995] han implementado un sistema formado por una red de este tipo con la ley de aprendizaje de retropropagación (badkpropagation) junto con un sistema de aprendizaje simbólico, para tratar de solucionar los dos grandes problemas de los sistemas booleanos, la imprecisión y mala especificación de las preguntas, y la indización incompleta e inconsistente [Larson 1992].

Brachman y Mcguinness [1988] utilizan una Máquina de Boltzman para hacer una recuperación conceptual que es simulada mediante un proceso de templecontro ladb El resultado aportado fue satisfactorio pese a que algunos aspectos relativos al proceso de temple y al ajuste de pesos debían ser reconsiderados. También se han utilizado en varias ocasiones para la expansión de preguntas. Un ejemplo es el proyecto Neurodoc [Lelu 1991, 1992], donde se ha representado todo el proceso documental como una red neuronal de tres capas, y se ha logrado la expansión de preguntas mediante una realimentación.

Se han empleado redes de Hopfield [1982] para asistir a los usuarios con las técnicas actuales, memorizando o generando tesauros, listas de encabezamiento, etcétera, para luego sugerir términos y rehacer la pregunta [Chen y Lynch 1992, Chen \&al. 1993, Chen 1995, Chen y Ng 1995, Chen \&. al. 1995, Chen et al. 1997]. Este tipo de tesauros generados automáticamente o "aproximadionesal espacioconeqp tual" [Chen \&. al. 1997], está siendo muy demandado en bases que tienen un contenido científico muy específico.

Jennings e Higuchi [1992] han diseñado una red neuronal para modelar usuarios, utilizada para aceptar 0 rechazar News de Usenet. Dicha red está formada por nodos (que representan palabras) con interconexiones entre ellos (que reproducen las relaciones entre los términos). Los nodos evolucionarán en función de los artículos leídos y rechazados; es decir, los nodos que representen palabras presentes en los artículos leídos, incrementarán su energía, y, al contrario, los que representen 
palabras de los rechazados la reducirán. Igualmente las relaciones entre términos aumentarán su peso si dichas palabras aparecen en los mismos artículos. Esto le permite al usuario añadir palabras claves en el momento de la recuperación, lo cual aumenta su peso, y variar el umbral, con lo que se puede cambiar la precisión y exhaustividad que se quiere obtener, con el fin de tener en cuenta sus intereses puntuales. También incorpora un cierto decaimiento de los pesos con el tiempo, que puede materializar el "dvidb”. Una vez que llega un artículo se calcula su energía (en función de los términos que contenga) y si supera el umbral se recupera. Este modelo de usuario va variando con el tiempo lentamente, en función de los artículos leídos y rechazados.

Kantor [1993] utiliza una red neuronal adaptativa en el programaANLI (Adaptative Network Library Interface) para incorporar recomendaciones aportadas por los usuarios. Se puede navegar por la red mediante una herramienta hipertextual contenida en la interfaz. Este sistema les permite a los usuarios el trabajocogperativopor adknadar compartiendo información.

Meghabghab [1994] utiliza redes neuronales en el diseño del INN, que sustituye a un experto en la negociación con el usuario para hacer búsquedas por materia. Emplea una red llamada ACN (Attentional Connectionist Network) para separar conceptos, agentes y relaciones, una base de conocimientos y un razonamiento analógico.

También se comienzan a explotar para el clustering, con este fin MacLeod y Robertson [1991] utilizan un modelo de red neuronal basado principalmente en ART (aunque, con algunas modificaciones). Se evalúa su rendimiento con las colecciones de Keen y Cranfield y se obtienen resultados similares a los algoritmos jerárquicos en cuanto a eficacia y algo mejor en cuanto a eficiencia. Sin embargo, tenemos que decir que utilizaron una representación documental muy pobre (casi la binaria) y no emplearon la posibilidad de ejecución en paralelo, pese a lo cual se consideraron satisfactorios sus resultados. Más tarde Muñoz [1994] utiliza un algoritmo híbrido entre FuzzyART y emediaspara hacer un clustering difuso de documentos. A simismo se ha llegado a hacer clustering de términos con el fin de poder llegar a la generación automática de tesauros [O akes y Taylor 1990].

Las redes de Rerrqurpagacón se han utilizado para la reducción dimensional de los espacios vectoriales documentales [Muñoz 1994] que, como ya hemos comentado, se puede lograr mediante el diseño de una red con muy pocas unidades ocultas. El inconveniente que tienen es el costoso aprendizaje que hay que llevar a cabo.

También se están utilizando redes de Kohonen para la generación de mapas topológics [Moya, Herrero y Guerrero, 1998b]. Una de las primeras aplicaciones de estos mapas en el procesamiento de texto fue la creación de representaciones topológicas sobre las relaciones semánticas entre palabras a partir de sus contextos [Ritter y Kohonen 1989].

El mismo Teuvo Kohonen dirige un grupo finlandés perteneciente al Centro de Investigación en Redes Neuronales de la Universidad Tecnológica de Helsinki que 
está utilizando este tipo de redes tanto para hacer clasificaciones de términos como de documentos:

* Ward Categry Map (mapa de categonías de palabras) [Honkela đ. al. 1995]: Se asigna un vector por ocurrencia de cada término que está dividido en tres partes iguales de noventa componentes cada una. La parte central corresponde a la codificación del término en cuestión, la primera parte a la del que le antecede y la última parte a la del que le sigue. Las codificaciones están formadas por noventa componentes entre cero y uno asignadas aleatoriamente a cada palabra. Estos vectores de 270 componentes son utilizados para entrenar una red de Kohonen que da lugar a un mapa en el que los nombres se agrupan en una zona, los verbos en otra, etcétera. Lo realmente innovador es la forma de incluir un pequeño contexto en la representación de cada palabra. En el caso de la lengua inglesa, por su estructura, se obtienen buenos resultados, que no tienen porqué ser extrapolables a otras lenguas.

* WEBSOM [K aski \&. al, 1996; Honkela \& al, 1996; Lagus \& al, 1996; Kohonen đ. al, 1999a, 1999b; Lagus \&. al, 1999; Lagus y Kaski, 1999]: Es un sistema pensado para clasificar un gran número de documentos, que se está aplicando a la Web. Para la representación de cada documento se siguen los siguientes pasos:

1. Se eliminan las palabras de alta y baja frecuencia, con el fin de reducir el procesamiento computacional y eliminar ruido.

2. Con estas palabras se genera un mapadecategríasdepalabras(Word category map).

3. Se construye un histograma para cada documento a partir de la respuesta del mapa de categorías de palabras a cada uno de los términos contenidos. De este modo la representación tendrá tantos componentes como elementos de proceso tenga el mapa de categorías de palabras.

Los vectores generados se utilizan para entrenar una red de Kohonen que los organiza temáticamente en dos dimensiones. Tiene una interfaz gráfica bastante cuidada que mediante la intensidad del color indica las distintas densidades de documentos.

Puede consultarse un prototipo de esto en internet [http:/ / websom.hut.fi/ websom/ ]. De este sistema es destacable la representación de los documentos, que al tener pocos componentes permite procesar un gran número de documentos con poco costo computacional.

Recientemente se ha desarrollado incluso un sistema que agiliza la búsqueda del cluster correspondiente a un documento o a una necesidad de información [Kaski 1999].

X ia Lin, investigador de la Universidad de Kentucky utiliza estos mapas con el fin de generar una salida visulizadepara una determinada colección de documentos [Lin 
1995, 1997]. El proceso que se lleva a cabo con los documentos es el siguiente:

1. Se construye de una lista que incluya todos los términos que aparecen en los títulos y resúmenes de todos los documentos de la colección.

2. Se suprimen los términos irrelevantes a partir de una lista de palabras vacías.

3. Se transforman los términos en la raíz mediante un algoritmo de stemming para reducir la lista.

4. Se eliminan términos de frecuencias altas y bajas.

5. Se construye un vector para cada documento, con tantos componentes como términos hayan quedado en la lista. Las componentes se generan de tres formas diferentes (dependiendo de la que se escoja se obtienen distintas visiones de la base):

a) Dígitos binarios (uno si el término correspondiente está en el documento y 0 si no está)

b) Pesos proporcionales a la frecuencia del término en el documento.

c) Pesos proporcionales a la frecuencia del término en el documento, e inversamente proporcionales al número de documentos en los que aparece el término.

Una vez que Lin ha generado los vectores correspondientes a todos los documentos, éstos son utilizados para entrenar la red de Kohonen. Terminado el entrenamiento se le asigna a cada neurona el término más cercano a su vector de pesos. De esta forma finalmente tenemos los documentos clasificados en una superficie dividida en una serie de zonas etiquetadas por términos. Ejemplos prácticos los podemos ver en la propia página personal de Lin [http:/ / www.ukv.edu/ xLin/ ].

Chen \&. al. [1998a] también utilizan un sistema muy parecido para crear una interfaz de acceso a la información y lleva a cabo una evaluación de la interfaz utilizando una serie de usuarios que lo comparan con un sistema clásico. Igualmente parecido es el sistema NeuroIsoc desarrollado para acceder a la base de datos del ISO c [Moya \&. al, 1999], aunque implica con una representación documental diferente basada en la coocurrencia de distintos descriptores.

En nuestro caso hemos comparado los resultados obtenidos mediante un sistema muy similar, con la clasificación ofrecida por los descriptores asignados a los documentos [Guerrero \& al. 2001]. El mismo sistema se utiliza tanto para el clustering, como para la representación topológica de términos y documentos [Guerrero, 1997].

Orwig, Chen y Nunamaker [1997] han utilizado mapas de este tipo para el procesamiento y clasificación de los comentarios de una "tometadeideas", y han llevado a cabo posteriormente una evaluación frente al algoritmo de Hopfield (también neuronal) [Chen \&. al. 1994] y a un experto humano. Tanto para una red como para la otra utiliza una representación documental binaria, para la cual se seleccionan los términos de mayor frecuencia (en el ejemplo aportado los 190 de mayor frecuencia). De este modo, cada comentario está representado por un vector que tiene tantos 
componentes como términos se utilizan para la representación, de los cuales serán uno los asociadas a los términos contenidos en el comentario y cero el resto. Estos vectores (tantos como comentarios han surgido en la "tomenta deideas") son utilizados para entrenar una red de Kohonen. Una vez terminado el entrenamiento se asigna cada documento al nodo cuyo vector de pesos es más cercano. Y a cada nodo se le asigna un término siguiendo un procedimiento similar al utilizado por Lin. La evaluación se lleva a cabo sobre la lista total de términos asignados a todos los nodos, tratando de examinar si la lista representa a todas las ideas aportadas. Para ello se utilizan los juicios de ocho expertos que generan las listas ideales así como una lista de asociaciones entre los términos. En la comparación que se hace de los tres sistemas (Kohonen, Hopfield y el humano) se obtienen los siguientes resultados:

* No existe diferencia significativa entre los tres sistemas en cuanto a la exhaustividad a la hora de generar la lista.

* En cuanto a la precisión el experto humano es significativamente superior a la red de Kohonen. No existen diferencias significativas entre la red de Hopfield y la de Kohonen.

* Son comparables los resultados de la asociación de la red de Kohonen (procedentes de la vecindad entre términos en la red) y el experto humano, y significativamente superiores ambos a los de la red de Hopfield.

D e esta evaluación se infiere que la red de Kohonen fue ligeramente inferior al experto humano (sólo en cuanto a precisión); sin embargo realizó su labor en seis minutos, mientras que el experto humano tardó más de cuarenta.

Campanario [1995] propone la utilización de redes de este tipo para analizar las relaciones entre revistas científicas de un determinado campo temático. Reyes \&. al. [2000] la utilizan para determinar los usos de revistas y las necesidades de información en los distintos departamentos de una universidad. Estudios similares también se han llevado a cabo mediante análisis de clusters, escalamiento multidimensional, análisis de componentes principales, etcétera [White y McCain 1998]. En todos los casos se genera una representación de las revistas a partir del Joumal Citation Repart (JCR) publicado por el Institute of ScientificInformation (ISI), que recoge las citas existentes entre diferentes títulos. Para cada caso se genera un vector con tantos elementos como títulos se vayan a analizar, y cada componente contendrá un valor dependiendo del número de citas de cada publicación. Con todos estos vectores se entrena una red que tiene más neuronas que títulos por analizar. Finalizado el entrenamiento se le asigna a cada neurona la revista cuyo vector se encuentra a una menor distancia del vector de pesos de la neurona, lo que da lugar al mapa de rdaciones Complementariamente se asocia cada título a la neurona ganadora y se genera el mapadedominios, que representa solamente las neuronas que se encuentran fuertemente ligadas a una determinada revista. Ambos mapas permiten, mediante el análisis del tamaño y la proximidad de las áreas, estudiar las relaciones existentes entre dos o más revistas científicas. 
Algunos autores consideran que las redes neuronales son un marco general que permite aplicar otras técnicas. D oszkocs \&. al. [1990] consideran que todos los modelos conexionistas pueden considerarse como sistemas de clasificación entrada/ salida. D e la misma manera, el clustering documental puede ser tomado como una clasificación del espacio documental en el mismo espacio [Willett 1988], la generación de tesauros como una clasificación del espacio de términos en sí mismo [Salton 1980] y la indización como una clasificación del espacio documental en el espacio de términos [Kwok 1990].

\section{Conclusiones}

Actualmente se han comenzado a aplicar técnicas de IA en documentación para superar los límites de los métodos clásicos. El campo en el que más se han utilizado es el de la recuperación de información, en gran medida por su formalización, el aumento de la información que hace inviables métodos tradicionales, y el impacto que tiene en la disciplina en su conjunto. Aunque no se han aplicado, al menos todavía, todas las técnicas de IA, sí se puede decir que se han aplicado técnicas de distintos tipos, como las técnicas de búsqueda (conocidas también como métodos débiles), los SS.BB.CC. y los modelos conexionistas.

Dentro de los SS.BB.CC. se han aplicado SS.EE. en el entorno profesional, como es lógico puesto que éstas son técnicas que tratan de competir con los expertos humanos. Su aplicación se centra en actividades muy formalizadas en las que se suele necesitar bastante experiencia, la cual, sin embargo, resulta difícil adquirir principalmente porque no son actividades muy comunes (como la catalogación de mapas, música, etcétera). También se han aplicado bases de conocimiento sobre el lenguaje que aunque han sido generadas con otros fines se pueden emplear para el análisis de textos.

Los AA.G G. son una de las técnicas de búsqueda (aunque guiadas por la función objetivo) que más notoriedad están alcanzando en la actualidad; y también se está empleando en documentación, concretamente en problemas de optimización. Se aplican a la indización, al clustering y principalmente a la retroalimentación por relevancia.

Y por último, se están utilizando modelos conexionistas para enfrentar problemas de aprendizaje y representación. Por un lado, se han generado grandes redes de palabras en las que se han tratado de representar las relaciones entre las palabras. Y por otro, se han utilizado RR.NN.AA. para problemas como la memorización de los términos de tesauros y sus relaciones, para el filtrado de mensajes, etcétera. Un tipo particular de redes que está tomando gran notoriedad en el área son los Mapas Auto-organizativos de Kohonen, que se emplean principalmente para visualizar las relaciones entre objetos, tanto términos como documentos, autores, revistas, etcétera. 


\section{Reconocimientos}

Este trabajo ha sido financiado por la Junta de Extremadura-Consejería de Educación Ciencia y Tecnología y el Fondo Social Europeo, como parte del proyecto de investigación IPR99A047.

\section{BIBLIOGRAFÍA}

AleXANDER,J.H. \&al. "Knowledge level engineering: ontological analysis". Procedings of AAAI 86, 963-968, 1986.

AMARI, C. "Characteristics of Random Nets of Analog Neuron-like Elements". IEEE TransadionsonSystens, Manand Cybemtics 2, 643-653, 1972.

A Mat I Noguera, Nuria. DoamentadónCientíficayNuevas Temdoǵasdela Informaión Madrid: Pirámide, 1989.

AnderSO N, J.A.; Silverstein, J.; Ritz, S.; Jones, R. "Distinctive features, categorical perception and probability learning: some applications on a neural model". Psychdogical Review 84, 413-451, 1977.

ARD IS, S. Online Patent "Searching: Guided by an Expert System". Online, 14(2), 56-62, ISSN 0146-54-22, 1990.

BELEW, R.K. "Adaptative Information Retrieval". Proceedingsof theAsciation forComputingMachineySpeial Interes GrouponInfomationRerieal (ACM SIGIR) 12thA mual Intemational Confereneon ReserthandDedopmentin Infomation Renieal, 25-28 de junio de 1989, Cambridge, MA., Nueva York, NY:ACM, Inc., 11-20, ISBN 0-89791-321-3, 1989.

Brachman, R.J.; Mcguinness, D.L. "Knowledge Representation, Connectionism, and Conceptual Retrieval". 11th International Conference on Research \& D evelopment in Information Retrieval; 13-15 de junio 1988; G renoble Francia, 161-174. Nueva Y ork, NY:Assocar tionforComputingMadiney, ISBN 2-7061-0309-4, 1988.

BRO OKS, H. "Expert Systems and Intelligent Information Retrieval". InformationProcessingandManagenet, 23(4), 367-382, ISSN 0306-4573, 1987.

BRUANDET, M.F. "O utline of a knowledge base model for an intelligent information retrieval system". Information Processing and Managenent, 25, 89-115, ISSN 0306-4573, 1989.

Buchanan, B. G.; Sutherland, G. L.; Feigenbaum, E. A. "Heuristic DEND RAL: a program for generating explanatory hypotheses in organic chemistry". In: Meltzer, B.; Michie, D .; Swann, M. (Eds.), Madime Intelligene4, Edinburgh, Scotland: Edinburgh University Press, 209254, 1969. 
Bush, V. "As We May Think". AtlanticMonthly, 176(1), 101-108, ISSN 0004-6795, 1945.

CAMPANARIO, J. M. "Using neural networks to study networks of scientific journals". Säetontrics 33(1), 23-40, 1995.

Cordón, O.; Moya, F.; Zarco, M.C. "Breve estudio sobrela aplicación de los algoritmos genéticos ala recuperación de lainformación". IV Congeso ISKO (G ranada), 179-186, 1999.

Co RTEZ, E. M, Sang, C. P.; Seonghee, K. "The Hybrid Application of an Inductive Learning Method and a Neural Network for Intelligent Information Retrieval". Infomation ProcessingandManagement, 31(6), 789813, ISSN 0306-4573, 1995.

CosTa Carballo, Carlos da. Introdurción a la Infomática Doamental: Fundamentos Ténicos, PrádicosyJuńdicos Madrid: Síntesis, S.A., 1995.

CHEN, H.; Lynch, K.J. "Automatic construction of networks of concepts characterizing document databases". IEEE TransadionsonSystens, Man andCybemetics 22(5), 885-902, 1992.

---; Lynch, K.J.; Basu, K.; Ng, D. T. “G enerating, integrating, and activating thesauri for concept-based document retrieval". IEEE EXPERT, Speeial Seies on Artifical Intelligence in Text-based Infomation Systens 8(2), 25-34, 1993.

---; Hsu, P.; O rwig, R.; Hoopes, L.; Nunamaker, J. F. "Automatic concept classification of text from electronics meetings". Commications of the ACM, 37(10), 56-73, 1994.

---. "Machine Learning for Information Retrieval: Neural Networks, Symbolic Learning, and G enetic Algorithms". Jamal of theAmricanSoidyforInfomationSaience, 46(3), 194-216, ISSN 0002-8231, 1995b.

---; Ng, D. T. "An algorithmic approach to concept exploration in a large knowledge network (automatic thesaurus consultation): Symbolic branch-and-bound vs. connectionist Hopfield net activation". Jaumal of the Amrican Socidy for Information Science, 46 (5), 348-369, ISSN 0002-8231, 1995.

Chen, H.; Schatz, B. R.; Yim, T.; Fye, D. "A utomatic Thesaurus generation for an electronic community system". Joumal of theAmericanSoaeyforInfamationSaience, 46(3), 175-193, ISSN 0002-8231, 1995.

---; Ng, D. T.; Martinez, J.; Schatz, B. R. "A Concept Space Approach to Addressing the Vocabulary Problem in Scientific Information Retrieval: An Experiment on the Worm Community System". Jaumal of the Ameican Socidy for Information Sáere, 48(1), 17-31, ISSN 0002-8231, 1997. 
Chen, H.; Houston, A.; Sewell, R.; Schatz, B. "Internet browsing and searching: user evaluations of category map and concept space techniques". Joumal of the American Sociey for Information Sience 49 (7), 582-603, 1998a.

---; Chung, Y.; Ramsey, M. "A smart itsy bitsy spider for the web”. Jaumal of theAmeican Sociky for Infomation Saience 49(7), 604-618, 1998b.

---; Iyer, A. "A machine learning approach to inductive query by examples: an experiment using relevance feedback, ID 3, genetic algorithms, and simulated annealing". Joumal of the American Socity for Information Saience, 49(8), 693-705, 1998c.

Chiaramella, Y .; D efued, B. "A prototype of an intelligent system for information retrieval: IOTA". Infomation Processingand Management, 23, 285-303, ISSN 0306-4573, 1987.

Debrower, A; Jones, D.T. "Application of an Expert Systems to Collection D evelopment: D onation Processing in a Special Library". LibrarySatwareReview, 10(6), 384-389, ISSN 0742-5759, 1991.

Do SzKO CS, T. E. "Natural language processing in information retrieval". Joumal of theAmerican Sociky for Infomation Sience, 37, 191-196, ISSN 0002-8231, 1986.

D o szKo cs, Tamas E.; Reggia, James; Lin, Xia. "Connectionist Models and Information Retrieval". Annul Review of Infomation Saienceand Tehndogy, 25, 209-260, ISSN 0066-4200, 1990.

D OY LE, L. B. "Semantic Road Maps for Literature Searchers" . Jaumal of theAssociation for Computing Madiney, 8, 553-578, ISSN 0004-5411, 1961.

ELLIS, D. New horizons in infomation retrieval. Londres: The Library Asociation, 1990.

---. Progess and problems in infomation retrieal. Londres: The Library Asociation, 1996.

ERCEg OVAC, Z. "Research on Knowledge-Based D escriptive Catalogin of Cartographic Publications: An Experimental Advice-Giving System Mapper" . Los Angeles, CA: UCLA (Ph.D. dissertation), 1990.

EveritT, B. Clusteranalysis 2nd ed. New Y ork: Halsted Press, 1980.

FUKUSHIMA, K. Cognitron: "A self-organizing multilayered neural network”. Bidogial Cybemetics, 20, 121-136, 1975.

FUKUSHIMA, K. Neocognitron: "A self-organizing neural network model for a mechanism of pattern recognition unaffected by shift in position". Bidogial Cybarmetics 36, 193-202, 1980. 
Gerber, B. ORFEO : "An Expert Reference Advisor for O pera". Library SoftwareReiien, 11(3), 8-11, ISSN 0742-5759, 1992.

GolDBERG, D .E. "G enetic algoritms in search, optimization and machine learning". Reding MA:Addison-Wesley, 1989.

Gómez, A.; Juristo, N.; Montes, C.; Pazos, J. Ingerieńa dd Conoimiento Madrid: Centro de Estudios Ramón A reces, S. A., 1997.

GORD ON, M.D. "A learning algorithm applied to document description". Procedings of the eighth annual intemational ACM/ SIGIR conferenceon reserchanddadqumentininformationrenieval. Montreal, 179-185, 1985.

GORD ON, M.D. "Probabilistic and genetic algorithms for document retrieval". CommmicationsofACM, 31(10), 1208-1218, 1988a.

GORD ON, M.D. "The necessity for adaptation in modified boolean document retrieval systems". Infomation Processingand Management, 24(3), 339-347, 1988b.

GORD ON, M.D. "User-based document clustering by redescribing subject descriptors with a genetic algorithms". Jaumal of theAmerican Soddyfor Information Sáence42(5), 311-322, 1991.

G ROSSBERG, S. "A prediction theory for some nonlinear functional-difference equation". Joumal of Mathematical A nalysisandApplications, 21, 643-694, 1968.

G Ro SSBERG, S. "Neural expectation: Cerebellar and retinal analogs of cells fired by learnable or unlearned pattern classes". Kybomeik, 10, 4957, 1972.

G Ruber T. "Toward Principles for the Design of Ontologies Used for Knowledge Sharing”. Technical Report KSL-93-04, Knowledge Systems Laboratory, Stanford University, CA, 1993.

GueRrERO, V. "Redes Neuronales aplicadas a las Técnicas de Recuperación D ocumental". Tesis doctoral, Universidad de G ranada, 1997.

Guerrero Bote, V.P.; Lozano Tello, A. "Vínculos entre las O ntologías y la Biblioteconomía y Documentación". Ađas del congreso EOCONSID’99, 25-31, 1999.

Guerrero, V.; Moya, F; Herrero V. "D ocument Organization using Kohonen's Algorithm". Information Processing and Management, (en prensa), 2001.

GunNing, K. "The Intelligent Reference Information System: The Effect on Public Services of Implementing a CD-ROM LAN and Expert System". LibraryAdministration andManagenent, 6(3), 146-153, ISSN 0888-4463, 1992. 
HAAS, S.W. "Natural Language Processing: Toward Large-Scale Robust Systems". Annual Reiewof Infomation Saienceand Techndogy, 31, 83119, ISSN 0066-4200, 1996.

HANFmAN, D . AquaRef: "An Expert Advisory System for Reference Support". RefereneLibraian, 23, 133-133, ISSN 0276-3877, 1989.

HAYEs-Roth, F.; Waterman, D.A.; Lenat, D. "An Overview of Expert Systems". In: Hayes-Roth, F.; Waterman, D.A.; Lenat, D. (Eds.), BuildingExpet Systems, Reading MA: Addison-Wesiey; 1983, 3-29. ISBN 0201-10686-8, 1983.

---; Wecker, J. "Knowledge Systems: An Introduction". LibraryHi Teeh 10 (1-2), 15-32, ISSN 0737-8831, 1992.

HECHT Nielsen, R. "Neurocomputing: Picking the Human Brain”. IEEE Spetum 25, 36-41, 1988.

HILERA, J. R.; Martínez, V. J. Reelesnerronalesartificiales, fundanentos, moddos yaplicadones Madrid: RAMA, 1995.

Ho LLAND, J.H. Adaptation in Natural and Artificial Systems $2^{\text {nd }}$ ed. MIT Press, 1992.

HonKela, T.; Pulkki, V; Kohonen, T. "Contextual relations of words in Grimm tales, analysed by self-orgnizing map". Proceedings of Intemational ConfereneonArtifical Nerral Newakss ICANN-95, París: EC2 et Cie, 3-7, 1995.

---; Kaski, S.; Lagus, K.; Kohonen, T. Seff-arganizing maps of dbament colletions Alma, 1(2), 1996.

Ho PFIELD, J.J. "Neural Networks and Physical Systems with Emergent Collective Computational Abilities". Procedings of theNational Academyof Saienes 79, 2554-2558, 1982.

HO RNG, J.T.; Yeh, C-C. "Applying genetic algorithms to query optimization in document retrieval". Information ProcessingandManagement, 36, 737-759, 2000 .

JACOBS, P. S.; Rau, L.F. "Natural language techniques for intelligent information retrieval". Proceedings of theAsociation for ComputingMadinery Speial Interest Group on Information Renieval (ACM/ SIG IR) 11th Annual International Conference on Research and D evelopment in Information Retrieval, 88-99, Washington, D C: ACM, 1988.

---; Rau, L. F. SCISO R: "Extracting information from the online news". Communications of theACM, 33, 88-97, 1990.

JenningS, A.; Higuchi, H. "A Browser with a neural network user model". LibraryHi Teh, 10 (1-2), 77-93, ISSN 0737-8831, 1992. 
KANTOR, Paul B. "The Adaptative Network Library Interface: A Historical Overview and Interim Report". LibraryHi Teh 11(3), 81-92, ISSN 0737-8831, 1993.

KASKI, S.; Honkela, T; Lagus, K.; Kohonen, T. Creatingan arderindigtal libranies withsedf-arganizingmaps Alma, 1(2), 1996.

---. "Fast winner search for SOM-based monitoring and retrieval of high-dimensional data". Proceedings of the Ninth Intemational ConfereneonArtifical Neural Networks(ICANN99) (pp. 940-945). London : Institution of Electrical Engineers, 1999.

KOHONEN, T. "Self-O rganized formation of topologically correct feature maps". Bidogical Cybemetics 43, 59-69, 1982. Reimpreso en: Anderson, J.; Rosenfeld, E. (Eds.), Neurocomputing Foundations of Reserch, Cambridge, Massachusetts: M.I.T. Press, 1988.

--.. "An Introduction to Neural Computing". Nerral Neworks 1, 3-16, 1988.

---; Kaski, S.; Lagus, K.; Salojärvi, J.; Honkela, J.; Paatero, V.; Saarela, A. "Self organization of a massive text document collection". En: Oja, E.; Kaski, S. (Eds.) Kohonen Maps, 171-182. Amsterdam: Elsevier, 1999a.

---; Kaski, S.; Lagus, K.; Salojärvi, J.; Honkela, J.; Paatero, V.; Saarela, A. "WEBSOM - A novel SO M-based approach to free-text mining". Neral NeworksRerchCentre(NNRC) at Helsinki University of Technology (HUT). http:/ / websom.hut.fi/ websom/ , 1999b.

KRAFT, D.H. etal. "Genetic algorithms for query optimization in information retrieval: relevancefeedback". En: Sanchez, E.; Shibata, T.;Zadeh, L.A. (Eds.) Genticalgrithms and fizzy logic systems Soft computingperspetives, 155-173, 1997.

Kwo K, K. L. "A Neural Network for Probabilistic Information Retrieval". ACMSIGRFonm 23(1-2), 21-30, 1989.

---. "Application of Neural Network to Information Retrieval". IJCNN90, Intemational JaintConferenceonNerral Neworks 15-19 de enero de 1990, Washington, D .C., Hillsdale, NJ.: Lawrence Erlbaum Associates, 623-626, ISBN 0-8058-0776-4, 1990.

LAGUS, K.; Kaski, S.; Honkela, T; Kohonen, T. "Self-orgnizing maps of document collections: anew approach to interactive exploration". In: Simoundis, Evangelios; Han Jiawei; Fayyad, U. (Eds.), Proceedings of theSecondIntemational ConferenceonKnoweedgeDiscoveryandDataMining Menlo Park, 1996, 1(2), 238-243, 1996.

---; Honkela, T.; Kaski, S.; Kohonen, T. "WEBSOM for textual data mining”. Artifiaal IntellignneReiews 13 (5/ 6), 345-364, 1999. 
LAGUS, K.; Kaski, S. "Keyword selection method for characterizing text document maps". Procedings of the Ninth Intemational Conference on Artifiaal Nerral Neworks(ICANN99) (pp. 371-376). London : Institution of Electrical Engineers, 1999.

LARSO N, R. R. "Evaluation of advanced retrieval techniques in an experimental online catalog". Joumal of theAmeican Sociky for Infomation Saience 43(1), 34-53, ISSN 0002-8231, 1992.

LEBOWITZ, M. "An experiment in intelligent information systems”. Com muications of theACM, 31, 1483-1502, 1986.

---. "The use of memory in text processing". CommuicationsoftheACM, 31, 1483-1502, 1988.

LELU, A. "From data analyssis to neural networks: new prospects for efficient browsing through databases". Jaumal of Infomation Saience, 17, 1-12, ISSN 0165-5515, 1991.

---; Francois, C. "Information based on neural unsupervised extration of thematic fuzzy clusters". Fifth Intemational Conference of Nerral Net works and their Aplications, Nerro Nimes92, Nimes (Francia), 2-6 noviembre, 93-104, 1992.

LIN, Xia. "Searching and browsing on Map Displays". Proceedings of the 58th Annual Meeting of the American Society for Infomation Saience (ASIS'95), Chicago, 13-18, 1995.

---. "Maps Displays for Information Retrieval". Jaumal of theAmerican SocieyforInformationSaience, 48(1), 40-54, ISSN 0002-8231, 1997.

LÓ PEZ-Pujalte, C. "Algoritmos genéticos aplicados a la retroalimentación por relevancia”. Tesis D octoral, Facultad de Biblioteconomía y D ocumentación, Universidad de G ranada, 2000.

MACLEOD, K.J.; Robertson, W. "A neural algorithm for documental clustering". Infomation Processing and Management, 27(4), 337-346, ISSN 0306-4573, 1991.

MALSBURG, C. Seff-organization of orientation sensitiva cells in the striate cattex. Kybernetik, 14, 85-100, 1973.

MARTín-Bautista, M.J. "Modelos de computación flexible para la recuperación de información”. Tesis D octoral, E.T.S. de Ingeniería de Informática, Universidad de Granada, 2000.

---; Vila, M.A.; Larsen, H.L. "A fuzzy genetic algorithm approach to an adaptive information retrieval agent". Joumal of theAmericanSocietyfor Information Saience, 50(9), 760-771, 1999.

McCullo CH, W. S. ; Pitts, W. "A logical calculus of the ideas immanent in nervous activity". Bulleinof Mathematical Biqphsiss 5, 115-133, 1943. 
MCD ERMOTT, D . "Artificial intelligence meets natural stupidity". SIGART Newsletter, 57, 1982.

MeghabghaB, G eorge V.; Meghabghab, Dania B. INN: "An intelligent negotiating neural network for information systems: a design model". InfomationProcessingandManagement, 30(5), 663-685, ISSN 0306-4573, 1994.

MiCHALEWICZ, Z. Genticalguithms + data sturtures = exdution programs Berlin: Springer-Verlag, 1995.

MinSKY , M.; Papert, S. Peceptrons AnIntroductiontoComputational Geem erry. Cambridge, MA: M.I.T. Press, 1969.

Moya Anegón, F.; Herrero Solana, V.; Guerrero Bote, V. “Las aplicaciones de las Redes Neuronales Artificiales (RNA): a la recuperación de información". En: Baro i Queralt, J;; Cid Leal, P. (Eds.), AnuarioSOCADI deDoumentacóneInfomacón 147-164, Barcelona: Societat Catalana de D ocumentació i Informació (SO CAD I), 1998.

---; Herrero, V.; Guemero, V. “Virtual reality interface for accessing electronic information". LibraryandInformationResearchNens 22(71), 34-39, 1998.

---;Moscoso, P.; Olmeda, C.; Ortiz-Repiso, V.; Hemero Solana, V.; G uerrero Bote, V. NeuroISOC: "un modelo dered neuronal para la representación del conocimiento". En: López Huertas, M.J.; Fenández Molina, J.C. (Eds.). La representación y la arganizacoón dd comaimiento en sus distintas perspetivas su influenda en la reuperacón dela informacón Adas dd IV Congeso ISKO-España (EO CO NSID ’99) (pp. 151-156). G ranada: ISKO -España, 1999.

MuÑOz García, Alberto. "Redes Neuronales para la Organización Automática de Información en Bases D ocumentales" . Tesis D octoral, Universidad de Salamanca, 1994.

O AKES, Michael P.; Taylor, Malcom J. Clusteingof ThesaunsTemsUsing AdaptiveResonameThery, FuzzyCogitiveMapsandApproximateString MatdhingTehniques The University of Liverpool, 1990.

O RWIG, R.; Chen, H.; Nunamaker, J. F. “A G raphical, Self-O rganizing Approach to Classifying Electronic Meeting O utput" .Jaumal of theAmmicanSoidyforInformationSäene, 48(2), 157-170, ISSN 0002-8231, 1997.

PARROTT, J. R. "Simulation of the Reference Process, Part II: REFSIM, an Implementation with Expert System and ICAI Modes". Referene Libranan 23, 153-176, ISSN 0276-3877, 1989.

Poulter, A.; Morris, A.; D ow, J. LIS. "Profesionals as Knowledge Engineers". Annual Reiew of Infomation Saienceand Telndogy, 29, 305350, ISSN 0066-4200, 1994. 
RAGHAVAN, V.V.; Agarwal, B. “O ptimal determination of user-oriented clusters: an application for the reproductive plan". Gendicalgrithmsand their applications Proceedings of the second intemational conference on gentic alggithmsand their applications 241-246, 1987.

---; Birchard, K. "A clustering strategy based on a formalism of the reproductive process in natural systems". En: Procedings of thesecondintemational ACM SIGIR confereneoninformationretrieval. D allas, 10-22, 1979.

RAU, L.F. "Knowledge organization and access in a conceptual information system". InformationProcessingandManagenent, 23, 269-283, ISSN 0306-4573, 1987.

---;Jacobs, P. S.; Zemik, U. "Information extraction and text summarization using linguistic knowledge acquisition". Infomation Processingand Managemet, 25, 419-428, ISSN 0306-4573, 1989.

Reyes Barragán, M.J.; G uerrero Bote, V.P.; Pulgarín Guerrero, A.; Zapico Alonso, F. "Revistas científicas: determinación de necesidades y usos". Reista Españda deDoumentadón Científica, 23, 417-436, ISSN 02100614, 2000 .

RICH, E.; Knight, K. Intdignia Artifiaal. Madrid: McGraw-Hill, D. L., 1994.

RitTeR, H.; Kohonen, T. "Self-organizing semantic maps". Bidogical Cybemeics 61, 241-254, 1989.

ROBERTSON, A.M.; Willett, P. “Use of genetic algorithms in information retrieval". British Library. Research and D evelopment D epartment. BLRD Repat6201, 1995.

---; Willett, P. "An upperbound to the performance of ranked-output searching: optimal weighting of query terms using a genetic algorithm". Joumal of Doumentation52(4), 405-20, 1996.

RosenblatT, F. The Perceptron: "A probabilistic model for information storage and organization in the brain". Psychdogical Reiew 65, 386-408, 1958. Reimpreso en: Anderson, J.; Rosenfeld, E. (Eds.), Neurocmputing Faundations of Rearch 92-114, Cambridge, Massachusetts: M.I.T. Press, 1988.

RUSSE LL, S. J. ; Norvig, P. Intdigeniaartificial: uneffoquemodema México: Prentice Hall, 1996.

Salto N, G. "Automatic Term Class Construction Using Relevance - A Summary of Work in Automatic Pseudoclassification". InfomationPro cessingandManagenet, 16(1), 1-15, ISSN 0306-4573, 1980.

---. "A Blueprint for Automatic Indexing" . ACMSIGIRFonm16(2), 2238, 1981 . 
SÁNCHEZ, E.; Miyano, H.; Brachet, J. “O ptimization of fuzzy queries with genetic algorithms. A pplication to a data base of patents in Biomedical Engineering". VI IFSA Congess Sao Paulo, Brazil, vol II, 293296, 1995.

SAMUEL, A. L. "Some studies in machine learning using the game of checkers". IBMJaumal ofReserchandDevdqument, 3(3), 210-229, 1959.

---. "Some studies in machine learning using the game of checkers IIRecent progress". IBMJaumal of ReserchandDedqument, 11(6), 601617, 1967.

SCHOLTES, J. Artificial neural neworks for infomation retrieval in libranies context Luxembourg: European Commision (Libraries in the Information Society), 1995.

Scotт A. C. \& al. Practical Guide to Knowedge Aqquisition AddisonWesley, 1991.

SMEATON, A.F. "Progress in application of natural language processing to information retrieval tasks". Thecomputerjaumal, 35, 268-278, 1992.

Smith, L. C. "Artificial Intelligence and Information Retrieval”. Annual Reviewof InfomationSaienceandTechnogy, 22, 41-77, ISSN 0066-4200, 1987.

Smith, K. F. "POINTER vs. Using G overnment Publications: Where's the Advantage?”. Referelelibranian 23, 191-205, ISSN 0276-3877, 1989.

SMITH, M.P.; Smith, M. "The use of genetic programming to build boolean queries for text retrieval through relevance feedback". Jaumal of Infomation Saience, 23(6), 423-31, 1997.

SowA, J. F. "Conceptual structures: Information Processing in Mind and Machine”. Reading, MA: Addison-Wesley, ISBN 0-201-14472-7, 1984.

SPARCK Jones, K. AutomaticKeyuord Classification for Infomation Retieval. Londres, England: Butterworths; ISBN 0-208-01201-X, 1971.

---. "Information Retrieval”. In: Shapiro, S.C. (Ed.), Engdopedia of Artifical Inteligence 1, 419-421, Nueva York, NY: John Wiley \& Sons, ISBN 0-471-80748-6, 1987.

Stiles, H. E. "The Association Factor in Information Retrieval”. Jaumal of theAssodation for Computing Madiney, 8(2), 271-279, ISSN 00045411, 1961.

TAYLOR, W. K. "Electrical simulation of some nervous system functional activities". In: Cherry, E.C. (Ed.), Infomation Theory, 3, 314-328, Londres: Butterworth, 1956. 
UlBRICHT, C.; D orffner, G .; Lee, A. “Neural Networks for Recognizing Patterns in Cardiotocograms". Submitted to: Artificial Intelligence in Medicine, 1999.

VAn Der Besselaar, Peter; Leydesdorff, Loet. "Mapping Change in Scientific Specialties: A Scientometric Reconstruction of the D evelopment of Artificial Intelligence". Joumal of theAmeicanSoidyfor InformationSäence, 47(6), 415-436, ISSN 0002-8231, 1997.

VICKERY, A.; Brooks, H. PLEX US: "The Expert System for Referral". InformationProcessingandMamagement, 23(2), 99-117, ISSN 0306-4573, 1987.

VRajito RU, V. "Crossover improvement for the genetic algorithm in information retrieval". InformationProcessingandManagenent34(4), 405415, 1998.

WARNER, A.J. "Natural language procesing”. Annual Reviend Information SämeandTechndogy, 22, 79-108, ISSN 0066-4200, 1987.

Welbank, M. A Reiiewof KnonkeedgeAcquisitionTechniques for Expet Sys tems Martlesham Heath, England: Martlesham Consultancy Services, 1983.

White, H.; Lin, X.; McCain, K. "Two modes of automated domain analysis: multidimensional scaling vs. Kohonen feature mapping of information science authors". En: Mustafa el-Hadi, W.; Maniez, J.; Pollit, A. S. (Eds.), Structures and reations in knovkeeke organization : procedings of thefifth Intemational ISKO Conference, 25-29 August 1998, Lille, France Proceedings of the 5th International ISK O Conference. Würzberg: Ergon Verlag, 1998.

WID ROW, B.; Hoff, M. "Adaptative Switching Circuits". IREWESCON ConventionReord, parte 4, 96-104, 1960. Reimpreso en: Anderson, J.; Rosenfeld, E. (Eds.), Neurocomputing Fandations of Reserch, 126-134, Cambridge, Massachusetts: M.I.T. Press, 1988.

--.. "Generalization and information storage in networks of adaline neurons”. In: Yovitz, M. C.; Jacobi, G. T.; Goldstein, G. D. (Eds.), SdfOrganizingSystems 435-461, Washington D.C.: Sparta, 1962.

WILKINSO N, R.; Hingston P. "Using the cosine measure in a neural network for document retrieval". Proceedings of theA sociationfor ComputingMadiney Speial Intees GraponInformationRetiexal (ACM/ SIG IR) 14th Annual International Conference on Research and D evelopment in Information Retrieval, 202-210, 1991.

---; Hingston P. "Incoporating the Vector Space Model in a Neural Network Used for D ocumet Retrieval". LibraryHi Teh 10 (1-2), 69-75, ISSN 0737-8831, 1992. 
WiLLETT, P. "Recent trends in hierachic document clustering: a critical review". Infomation Processingand Management, 24(5), 577-597, ISSN 0306-4573, 1988.

YANG, J.J.; Korfhage, R. "Query modification using genetic algorithms in vector space models". Intemational Jaumal ofExpat Systems, 7(2), 165191, 1994.

Y U, C.T.; Wang, Y.T .; Chen, C.H. "Adaptative document clustering” . Pro ceetings of theighth annul ACMV SIGIR conferenceon resench and deveqpmentininfomationretrieval. Montreal, 197-203, 1985. 